\title{
THE TREATMENT OF ASSETS IN PENSION FUNDING
}

\author{
BY \\ M. Iqbal Owadally and Steven Haberman
}

\begin{abstract}
A recent survey of actuarial practitioners in North America shows that smoothedmarket actuarial asset values are commonly used in funding valuations of defined benefit pension plans. Four methods of calculating such values are reported in the actuarial literature but only qualitative descriptions of the methods are given. This paper provides mathematical descriptions of the "average of market", "weighted average", "deferred recognition" and "write-up" actuarial values. They are shown to be based on either arithmetic or exponential smoothing. Provided the same form of smoothing is used, the four methods are equivalent.
\end{abstract}

\section{KEYWORDS}

Actuarial valuation, assets, losses, smoothing.

\section{INTRODUCTION}

Actuaries perform various types of valuations on defined benefit pension plans. Solvency or minimum funding valuations are carried out in many jurisdictions in accordance with regulation or statute. The aim of a solvency valuation is to assess the ability of a pension plan to meet all liabilities in the event that the plan is wound up. The up-to-date fair market value of plan assets is used in solvency valuations and any other value would be irrelevant. Off-market asset values are also not meaningful for accounting valuations, when the economic cost to plan sponsors of providing pensions is being measured.

Only funding valuations are considered in this paper. The purpose of a funding valuation is to calculate a suitable contribution rate. One reason for funding pensions in advance is that contributions, from plan sponsor or members, can be planned, budgeted and invested over time. Actuarial funding methods are designed to organise an orderly and systematic funding of benefits. Contributions are in effect smoothed over time resulting in a regular and stable pattern of contributions. To this end, actuarial asset values, which are typically an average of market asset values over short intervals, are used to dampen volatility when calculating contribution rates. It is important to emphasise that 
an actuarial asset value is not a substitute for the market value of assets but is a device to spread the funding of pensions over time in a systematic way: see Ezra (1979, p. 40), Anderson (1992, p. 108) and Winklevoss (1993, p. 171) among others. In particular, investment decisions should be based on market values and not actuarial values (Ezra, 1979, p. 110).

The aim of this paper is to provide concise mathematical formulas for some of the actuarial asset values that are used by actuarial practitioners, and to show how they relate to each other. Section 2 sets out some simple notation which is used to describe the various actuarial asset values that are discussed in section 3. The "average of market" (section 3.1), the "weighted average" (section 3.2) the "deferred recognition" (section 3.3) and the "write-up" (section 3.4) are considered. The calculation of some of these values is described in words in the survey of the Committee on Retirement Systems Research, henceforth referred to as CRSR (2001).

\section{NotATION}

Cash flows in and out of a defined benefit pension fund consist of contributions paid in by plan members and by the plan sponsor, and benefits and expenses paid out. It is assumed here that cash flows occur at the start of discrete time intervals $[t, t+1]$. The market value of pension plan assets at time $t$ is denoted by $F_{t}$ and the net cash outgo from the fund at time $t$ is denoted by $C F_{t}$.

Funding valuations are carried out regularly with the aim of establishing a contribution rate. To this end, an actuarial liability is calculated based on a set of valuation assumptions concerning investment return, mortality rates, withdrawal rates, inflation, salary scales etc. The unfunded liability of the pension plan is the excess of actuarial liability over the market value of the plan assets. Actual experience generally deviates from actuarial valuation assumptions, resulting in experience gains or losses. Aitken (1994) describes gain and loss calculation in detail. A gain is a negative loss and henceforth we refer to losses only.

The overall loss may be decomposed as a sum of liability loss and asset loss. Asset losses emerge when actual investment returns deviate from the actuarial assumption about investment return; liability losses emerge when deviations from actuarial assumptions about mortality, withdrawal etc. occur.

The valuation assumption about the rate of return on plan assets is denoted by $i$ and is taken to be constant over time. Let $u=(1+i)$ and $v=(1+i)^{-1}$. The asset loss at time $t$ is denoted by $L_{t}$. The expected market value of plan assets at time $t$ based on the investment return valuation assumption at time $t-1$ is $u\left(F_{t-1}-C F_{t-1}\right)$. The actuarial asset loss is the unexpected decrease in the market value of plan assets based on the investment return valuation assumption (Aitken, 1994, p. 162):

$$
L_{t}=u\left(F_{t-1}-C F_{t-1}\right)-F_{t} .
$$

Suppose that $X_{t}$ denotes the value of the pension fund. $X_{t}$ can be either the market value or an actuarial asset value. Let $\mathcal{L}$ be the lag or backward shift 
operator such that $\mathcal{L}^{j} X_{t}=X_{t-j}$ where $j \in \mathbb{Z}$. The write-up operator $\mathcal{W}$ is then defined as follows:

$$
\mathcal{W} X_{t}=u\left(\mathcal{L} X_{t}\right)-u C F_{t-1} .
$$

The interpretation of $\mathcal{W} X_{t}$ is that it is the value of the pension fund at time $t-1$ written up to time $t$ allowing for interest and intermediate cash flows.

The asset loss defined in equation (1) may be expressed more concisely using the write-up operator as follows:

$$
L_{t}=(\mathcal{W}-1) F_{t}
$$

The write-up operator may be applied $j$ times $(j \in \mathbb{N})$ :

$$
\mathcal{W}^{j} X_{t}=u^{j} X_{t-j}-\sum_{k=1}^{j} u^{k} C F_{t-k} .
$$

Equation (4) is easily verified by repeated application of $\mathcal{W}$ to $X_{t}$ and may also be proven by induction. $\mathcal{W}^{j} X_{t}$ may be interpreted as the value of the pension fund at time $t-j$ written up to time $t$ allowing for interest and cash flows into and out of the pension fund. By convention, $\mathcal{W}^{0} X_{t}=X_{t}$, that is $\mathcal{W}^{0}=1$.

The loss $L_{t-j}$ at time $t-j$ may be expressed in terms of the market value of assets at time $t-j$ : from equation (3), $L_{t-j}=(\mathcal{W}-1) F_{t-j}$. But $L_{t-j}$ may also be expressed in terms of the market values at time $t-j$ written up to time $t$ :

$$
L_{t-j}=v^{j}\left(\mathcal{W}^{j+1}-\mathcal{W}^{j}\right) F_{t}
$$

$L_{t-j}$ is the difference in the written-up asset values in the interval $[t-j-1, t-\mathrm{j}]$, discounted back over $j$ time intervals.

Proof of equation (5): Using equation (4), the right hand side of equation (5) is $v^{j}\left[u^{j+1} F_{t-j-1}-\sum_{k=1}^{j+1} u^{k} C F_{t-k}-u^{j} F_{t-j}+\sum_{k=1}^{j} u^{k} C F_{t-k}\right]=u F_{t-j-1}-u C F_{t-j-1}-$ $F_{t-j}$ which is equal to $(\mathcal{W}-1) F_{t-j}=L_{t-j}$.

Two smoothing operators on the market value $F_{t}$ of plan assets are also defined: an exponential smoothing operator $\mathcal{E}$ with smoothing parameter $\lambda$ (where $0 \leq \lambda<1$ ):

$$
\mathcal{E} F_{t}=(1-\lambda) \sum_{j=0}^{\infty}(\lambda \mathcal{W})^{j} F_{t}
$$

and an arithmetic smoothing operator $\mathcal{A}$ with averaging period $n$ (where $n \in \mathbb{N}$ ):

$$
\mathcal{A} F_{t}=\frac{1}{n} \sum_{j=0}^{n-1} \mathcal{W}^{j} F_{t}
$$

The weights or coefficients of $\left\{F_{t}\right\}$ in both smoothing operators sum to unity, that is, $\sum_{j=0}^{\infty}(1-\lambda) \lambda^{j}=1$ and $\sum_{j=0}^{n-1} \frac{1}{n}=1$. When $\lambda=0, \mathcal{E} F_{t}=F_{t}$; when $n=1, \mathcal{A} F_{t}=F_{t}$. 
We interpret $\mathcal{E} F_{t}$ as the actuarial value of pension plan assets at time $t$ based on an exponential smoothing of market values. Likewise, $\mathcal{A} F_{t}$ is the actuarial value of pension plan assets at time $t$ based on an arithmetic averaging of market values. Our aim is to show that $\mathcal{A} F_{t}$ and $\mathcal{E} F_{t}$ correspond to commonly used definitions of actuarial asset values.

\section{Smoothed-Market Actuarial Asset Values}

Various types of actuarial asset values are used in practice. CRSR (2001) reports that about $30 \%$ of US defined benefit pension plans with funds whose market value is in excess of US\$1m use smoothed-market actuarial values. By contrast, discounted cash flow values and book values are used sparingly. Four smoothed-market actuarial values are described hereunder.

\subsection{Average of Market}

CRSR (2001) defines in words the "average of market" actuarial asset value:

"A preliminary asset value is developed as the average of the current Fair Market Value and one or more Adjusted Fair Market Values (AFMV) from previous years. The AFMV for each prior year is developed by adjusting that year's Fair Market Value to the valuation date, by adjusting contributions, subtracting benefits paid (and possibly expenses) and further adjusting by certain specific items of investment experience."

The "average of market" value may involve either exponential or arithmetic smoothing. We consider the arithmetic smoothing form first and we show that it is equal to $\mathcal{A} F_{t}$ :

$$
\begin{aligned}
\mathcal{A} F_{t} & =\frac{1}{n}\left[F_{t}+\sum_{j=1}^{n-1}\left(u^{j} F_{t-j}-\sum_{k=1}^{j} u^{k} C F_{t-k}\right)\right] \\
& =\frac{1}{n} \sum_{j=0}^{n-1} u^{j} F_{t-j}-\frac{1}{n} \sum_{j=1}^{n-1}(n-j) u^{j} C F_{t-j} .
\end{aligned}
$$

Proof of equation (8a): From the definition of the arithmetic smoothing operator in equation (7), $\mathcal{A} F_{t}=\frac{1}{n}\left[F_{t}+\sum_{j=1}^{n-1} \mathcal{W}^{j} F_{t}\right]$. Now, $\mathcal{W}^{j} F_{t}$ is the quantity called the "adjusted fair market value" for the fund for time $t-j$ (for $j \geq 1$ ) by CRSR (2001) in the quote above. $\mathcal{W}^{j} F_{t}$ may be rewritten using equation (4) to give the summand in the outer sum on the right hand side of equation (8a).

Equation (8b) follows in a straightforward fashion from equation (8a) and shows explicitly that an average over $n$ years of the market values of the plan assets is being calculated, with allowance for both interest and cash flows. The "average of market" actuarial value with arithmetic smoothing is also known as a "moving average of market". 
The "average of market" value may also be construed with exponential smoothing, in which case we show that it is identical to $\mathcal{E} F_{t}$ :

$$
\begin{aligned}
\mathcal{E} F_{t} & =(1-\lambda) F_{t}+(1-\lambda) \sum_{j=1}^{\infty} \lambda^{j}\left(u^{j} F_{t-j}-\sum_{k=1}^{j} u^{k} C F_{t-k}\right) \\
& =(1-\lambda) \sum_{j=0}^{\infty}(\lambda u)^{j} F_{t-j}-\sum_{j=1}^{\infty}(\lambda u)^{j} C F_{t-k}
\end{aligned}
$$

Proof of equation (9a): Using the definition of the exponential smoothing operator in equation (6), $\mathcal{E} F_{t}=(1-\lambda) F_{t}+(1-\lambda) \sum_{j=1}^{\infty}(\lambda \mathcal{W})^{j} F_{t}$. Again, $\mathcal{W}^{j} F_{t}$ may be replaced using equation (4) yielding equation (9a).

Equation (9b) follows from equation (9a) and demonstrates that the market values of the plan assets are being exponentially smoothed, with allowance for both interest and cash flows.

\subsection{Weighted Average}

A "weighted average" actuarial value is also commonly used (Winklevoss, 1993, p. 172). We show that it is equal to $\mathcal{E} F_{t}$ and is identical to the "average of market" value with exponential smoothing. It averages the current market value of plan assets $\left(F_{t}\right)$ and the written-up actuarial value from the last year $\left(\mathcal{W} \mathcal{E} F_{t}\right)$, with weights of $1-\lambda$ and $\lambda$ respectively:

$$
\mathcal{E} F_{t}=(1-\lambda) F_{t}+\lambda \mathcal{W} \mathcal{E} F_{t}
$$

Proof of equation (10): All summations are over $j \in[0, \infty)$. Making use of the definition of the exponential smoothing operator in equation (6), the operations on the right hand side of equation (10) may be written as $1-\lambda+\lambda \mathcal{W}_{\mathcal{E}}=$ $1-\lambda+\lambda \mathcal{W}(1-\lambda) \Sigma(\lambda \mathcal{W})^{j}$. Factoring $(1-\lambda)$ gives $(1-\lambda)\left(1+\Sigma(\lambda \mathcal{W})^{j+1}\right)$ which simplifies to $(1-\lambda) \Sigma(\lambda \mathcal{W})^{j}$ which is the $\mathcal{E}$ operator that appears on the left hand side of equation (10).

\subsection{Deferred Recognition or Adjusted Market}

Another common actuarial asset value is the "deferred recognition" value. CRSR (2001) defines it as follows:

"Under this method, only a portion of investment experience is recognised in the current year. A preliminary asset value is developed by subtracting (or adding) a portion of previously unrecognised gains (or losses) from the current Fair Market Value."

The "deferred recognition" method may contain either exponential smoothing or arithmetic smoothing. First, we consider "deferred recognition" with exponential 
smoothing and show it to be equivalent $\mathcal{E} F_{t}$ and thus to the "average of market" with exponential smoothing:

$$
\mathcal{E} F_{t}=F_{t}+\sum_{j=0}^{\infty} \lambda^{j+1} u^{j} L_{t-j}
$$

Proof of equation (11): All summations are over $j \in[0, \infty)$ unless stated otherwise. Replace $L_{t-j}$ from equation (5) into the right hand side of equation (11) to obtain

$$
\begin{aligned}
& F_{t}+\sum \lambda^{j+1}\left(\mathcal{W}^{j+1}-\mathcal{W}^{j}\right) F_{t}=F_{t}+\sum_{j=1}^{\infty}(\lambda \mathcal{W})^{j} F_{t}-\lambda \sum(\lambda \mathcal{W})^{j} F_{t} \\
& =\sum(\lambda \mathcal{W})^{j} F_{t}-\lambda \sum(\lambda \mathcal{W})^{j} F_{t}=(1-\lambda) \sum(\lambda \mathcal{W})^{j} F_{t}=\mathcal{E} F_{t},
\end{aligned}
$$

by virtue of the definition of the $\mathcal{E}$ operator in equation (6).

The "deferred recognition" method of equation (11) may be understood intuitively as follows. A loss is recognised as an infinite sum of exponentially declining amounts. That is, a unit loss is smoothed by recognising $\left\{(1-\lambda) \lambda^{0},(1-\lambda) \lambda^{1}\right.$, $\left.(1-\lambda) \lambda^{2}, \ldots\right\}$ together with interest, in successive years and in perpetuity. For a unit loss that emerged $j$ years ago, the total loss that has been recognised is $1-\lambda^{j+1}$ (along with interest). The remainder $\left(\lambda^{j+1}\right.$ along with interest) is deferred and thus added to the current market value.

The "deferred recognition" method with exponential smoothing is equivalent to the "average of market" with exponential smoothing and to the "weighted average", the only difference being that it is computed in terms of asset losses. In fact, the "deferred recognition" method is sometimes called an "adjusted market" method because the actuarial value of plan assets is taken to be the current market value $\left(F_{t}\right)$ together with an adjustment equal to a fraction $(\lambda)$ of the difference between the written-up actuarial value from last year $\left(\mathcal{W E} F_{t}\right)$ and the market value $\left(F_{t}\right)$ :

$$
\mathcal{E} F_{t}=F_{t}+\lambda\left(\mathcal{W} \mathcal{E} F_{t}-F_{t}\right) .
$$

Proof of equation (12): Rearranging equation (10) yields equation (12).

Another version of the "deferred recognition" method employs arithmetic smoothing, and we show that it is equivalent to $\mathcal{A} F_{t}$ and to the "average of market" value with arithmetic smoothing:

$$
\mathcal{A} F_{t}=F_{t}+\sum_{j=0}^{n-2} \frac{n-1-j}{n} u^{j} L_{t-j}
$$

Proof of equation (13): All summations are over $j \in[0, n-2]$ unless stated otherwise. Replace $L_{t-j}$ from equation (5) into the right hand side of equation (13) to obtain

$$
F_{t}+\sum \frac{n-1-j}{n}\left(\mathcal{W}^{j+1}-\mathcal{W}^{j}\right) F_{t}=F_{t}+\sum_{j=1}^{n-1} \frac{n-j}{n} \mathcal{W}^{j} F_{t}-\sum \frac{n-j}{n} \mathcal{W}^{j}+\sum \frac{1}{n} \mathcal{W}^{j} F_{t}
$$


$=\frac{1}{n} \mathcal{W}^{n-1} F_{t}+\frac{1}{n} \sum \mathcal{W}^{j} F_{t}=\frac{1}{n} \sum_{j=0}^{n-1} \mathcal{W}^{j} F_{t}=\mathcal{A} F_{t}$,

the last equality following from the definition of the $\mathcal{A}$ operator in equation (7). $\square$

In the deferred recognition method of equation (13), a fraction $1 / n$ of each asset loss over the past $n-1$ years is recognised, while the rest is deferred. The deferred portions (along with interest earned) are added to the current market value of assets. Compare exponential smoothing (equation (11)) with arithmetic smoothing (equation (13)): losses are deferred in perpetuity under the former.

\subsection{Write-up with Adjustment}

CRSR (2001) also states that pension actuaries use an actuarial value called the "write-up with adjustment":

"A preliminary asset value is developed by bringing forward the prior year's actuarial asset value, adding contributions, subtracting benefit payments (and possibly expenses) and increasing this result with assumed earnings. $(\cdots)$ This preliminary asset value could be subject to certain other adjustments to develop a final asset value. The adjustment ( $\cdots)$ might include a partial adjustment towards Fair Market Value."

One form of the "write-up" method contains exponential smoothing and we show that it is equivalent to $\mathcal{E} F_{t}$ and to all the methods described above where exponential smoothing was employed:

$$
\mathcal{E} F_{t}=\mathcal{W E} F_{t}+(1-\lambda)\left(F_{t}-\mathcal{W E} F_{t}\right) .
$$

$\mathcal{W E} F_{t}$ is the written-up actuarial value of assets from the previous year (or the "preliminary asset value" in the quote above from CRSR (2001)) which is then adjusted by recognising a fraction of the difference between the market value and the written-up actuarial value.

Proof of equation (14): This equation follows by rearranging equation (10).

Another form of the "write-up" method with exponential smoothing calculates the adjustment directly in terms of the asset losses, but is, of course, identical to the form given in equation (14):

$$
\mathcal{E} F_{t}=\mathcal{W} \mathcal{E} F_{t}-\sum_{j=0}^{\infty}(1-\lambda)(\lambda u)^{j} L_{t-j}
$$

A unit loss is smoothed by recognising $\left\{(1-\lambda) \lambda^{0},(1-\lambda) \lambda^{1},(1-\lambda) \lambda^{2}, \ldots\right\}$ together with interest, in successive years and in perpetuity. In this method, the written-up actuarial value is therefore adjusted by recognising portions of past losses.

Proof of equation (15): Observe that $F_{t}-\mathcal{W E} F_{t}=-\sum_{j=0}^{\infty}(\lambda u)^{j} L_{t-j}$ by comparing equations (11) and (12). Hence rewrite the second term on the right hand side of equation (14) in terms of losses to get equation (15). 
The write-up method is also used with arithmetic averaging:

$$
\mathcal{A} F_{t}=\mathcal{W} \mathcal{A} F_{t}-\frac{1}{n} \sum_{j=0}^{n-1} u^{j} L_{t-j}
$$

$\mathcal{W} \mathcal{A} F_{t}$ is the written-up actuarial value of assets with arithmetic smoothing from the previous year, that is, it is the "preliminary asset value" in the quote above from CRSR (2001). $\mathcal{W} \mathcal{A} F_{t}$ is adjusted downwards by the sum of recognised portions of previous losses. See Peat Marwick (1986, p. 25) for an explicit example where this method is used in conjunction with accounting valuations under Financial Accounting Standard No. 87. The write-up method of equation (16) is of course equivalent to all the methods discussed previously where arithmetic smoothing was used.

Proof of equation (16): All summations are over $j \in[0, n-1]$ unless stated otherwise. Applying the write-up operator (equation (2)) to $\mathcal{A} F_{t}$ in equation (7) gives $\mathcal{W} \mathcal{A} F_{t}=\frac{1}{n} \sum \mathcal{W}^{j+1} F_{t}$. Using equation (5), $\frac{1}{n} \sum u^{j} L_{t-j}=\frac{1}{n} \Sigma\left(\mathcal{W}^{j+1}-\mathcal{W}^{j}\right) F_{t}=$ $\frac{1}{n}\left(\mathcal{W}^{n}-1\right) F_{t}$. Hence the right hand side of (16) is $\frac{1}{n} \sum \mathcal{W}^{j+1} F_{t}-\frac{1}{n} \mathcal{W}^{n} F_{t}+\frac{1}{n} F_{t}=$ $\frac{1}{n} \sum \mathcal{W}^{j} F_{t}=\mathcal{A} F_{t}$, where the last equality follows from the definition of $\mathcal{A}$ in equation (7).

\subsection{Equivalence}

It was shown above that the four actuarial asset values are equivalent, provided they incorporate the same form of smoothing (that is, arithmetic or exponential). $\mathcal{E} F_{t}$ and $\mathcal{A} F_{t}$ in equations (6) and (7) respectively are generic forms of smoothed-market actuarial asset values. The practical implementation of these methods requires initialization values. For example, in the "average of market" with arithmetic smoothing (equation (8b)), past market values of plan assets may not be available and may be replaced by the current market value. Likewise, in exponential smoothing, only a finite amount of past data on losses or market values are available (equations (9b) and (11)). Although the methods are equivalent, practical differences in the initial conditions may result in different values in the short term.

\section{ConcLusion}

An actuarial asset value is not a substitute for the market value of assets but is used to moderate volatility and devise a stable pattern of contributions. Four smoothed-market actuarial values were described. They are frequently used by actuaries according to a comprehensive survey by the Committee on Retirement Systems Research (CRSR, 2001). Mathematical formulae were given for the four methods, as opposed to the qualitative descriptions found elsewhere. It was shown that the methods have a common exponential or arithmetic smoothing methodology. Past and present market values are smoothed but explicit allowance for interest and for intermediate cash flows is made. Except 
for the form of smoothing being either exponential or arithmetic, and except for initialization procedures, the four methods were shown to be equivalent.

The mathematical descriptions of the actuarial asset values should help in understanding and improving pension funding methods. Work on further comparison between arithmetic and exponential smoothing is ongoing. The choice of a suitable averaging period $n$ and exponential smoothing parameter $\lambda$ (Owadally \& Haberman, 2003) is also being investigated.

\section{REFERENCES}

Aitken, W.H. (1994) Pension Funding and Valuation. Actex Publications, Winsted, Connecticut. Anderson, A.W. (1992) Pension Mathematics for Actuaries, 2nd ed. Actex Publications, Winsted, Connecticut.

CRSR (2001) Survey of asset valuation methods for defined benefit pension plans. Pension Forum, 13(1), 1-49. Society of Actuaries, Schaumburg, Illinois.

Ezra, D.D. (1979) Understanding Pension Fund Finance and Investment. Pagurian Press, Toronto, Canada.

OwadAlly, M.I. and Haberman, S. (2003) Exponential smoothing methods in pension funding. IMA Journal of Management Mathematics 14(2), 129-143.

Peat Marwick (1986) Interpretation of Pension Statements. Note 461-32-87, Society of Actuaries, Schaumburg, Illinois.

Winklevoss, H.E. (1993) Pension Mathematics with Numerical Illustrations, 2nd ed. University of Pennsylvania Press, Philadelphia, Pennsylvania.

\section{Iqbal Owadally and Steven Haberman}

Faculty of Actuarial Science and Statistics

Cass Business School

City University

106 Bunhill Row, London EC1 Y 8TZ, England

Email:iqbal@city.ac.uk 